\title{
Continuous endobronchial suctioning for refractory post lobectomy lung atelectasis: a case report
}

\author{
Vasileios Kouritas ${ }^{1}$, Neisha Ross ${ }^{2}$, Andrey Bilyy ${ }^{2}$, John Hogan ${ }^{2}$, Moondi Parvez ${ }^{3}$, Jakub Kadlec ${ }^{2}$, \\ Waldemar Bartosik', Filip Van Tornout ${ }^{2}$ \\ ${ }^{1}$ Department of Thoracic Surgery, Norfolk and Norwich University Hospital, Norwich, UK; ${ }^{2}$ St. George's Medical School, Grenada; ${ }^{3}$ Department of \\ Anesthesiology and Critical Care, Norfolk and Norwich University Hospital, Norwich, UK \\ Correspondence to: Mr. Vasileios Kouritas, CTh, MD, PhD. Department of Thoracic Surgery, Norfolk and Norwich University Hospital, Colney \\ Lane, NR4 7UY, Norwich, UK. Email: vasileios@doctors.org.uk.
}

\begin{abstract}
The presentation of post lung resection atelectasis can vary between simple atelectasis and total lung collapse i.e., "white - out", making its treatment demanding in many occasions. We herein present the technique of continuous suctioning of the right upper lobe (RUL) by positioning a suction catheter inside the right upper lobe bronchus (RULB) through a tracheostomy in a sedated patient. This technique was used in the case of a 70-year-old patient who underwent a complicated redo thoracotomy and right lower lobectomy for lung cancer after a previous middle lobectomy via double thoracotomy for similar pathology. He had a significant ankylosis spondylitis past medical history with bamboo spine treated with long term high doses of steroids and methotrexate. Post redo surgery he developed respiratory failure with a radiologically significant RUL collapse, i.e., a "white-out", of the operated side which was refractory to usual conservative or bronchoscopic treatment. As a last resort, and in an effort to avoid high risk pneumonectomy, the patient was sedated, and a suction catheter was left inside the RULB under direct bronchoscopic guidance. This allowed the secretions inside the airways to be cleared, giving the remaining upper lobe infection time to subside, protected the stump from infective secretions and blind suctioning and led to avoidance of a high-risk pneumonectomy. The upper lobe cleared up from its collapse and patient's discharge from high dependency unit was achieved. This described maneuver can be useful in refractory cases of atelectasis when other measures have failed, in borderline patients or in patients where further surgery is technically cumbersome.
\end{abstract}

Keywords: Lobectomy; refractory; atelectasis; catheter; case report

Submitted May 10, 2020. Accepted for publication Nov 15, 2020.

doi: 10.21037/atm-20-3839

View this article at: http://dx.doi.org/10.21037/atm-20-3839

\section{Introduction}

Pulmonary infectious complications are common after lung resections mainly with the form of atelectatic lung collapse and pneumonia (1). Development of such complications may lead to increased length of stay, length of stay in intensive care unit as well as increased 30-day and 90day mortality (2). The severity of these complications is influenced by many factors i.e., their extent and mechanism of development, patient vulnerability, the compensatory reserves and others (3).
Conservative treatment of these complications usually includes antibiotics, use of mycolytics for reduced secretion volume, aggressive physiotherapy etc. $(1,3)$. In some cases, more aggressive interventions may be needed i.e., non-invasive ventilation and direct airway suctioning (with bronchoscopy or a tracheostomy) $(1,3)$. There are, however, rare cases in which the collapse persists, despite all the aforementioned measures, leading to the need for improvisation in an effort to avoid further surgery, which in borderline patients can be detrimental $(4,5)$.

We herein present the technique of continuous 
Page 2 of 6

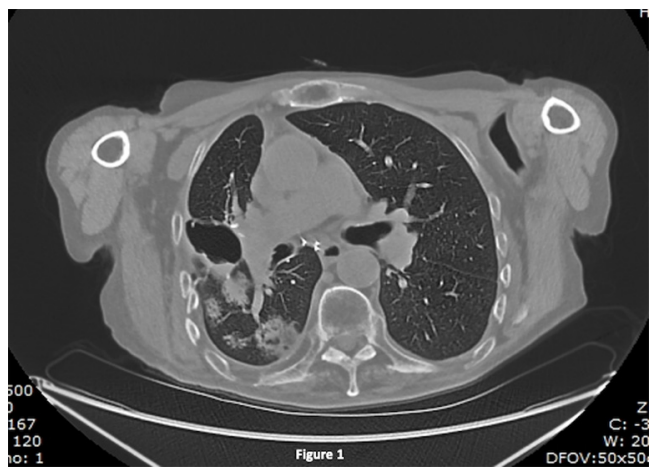

Figure 1 Preoperative CT chest scan of the patient showing the lesions in the right lower lobe. It also shows the space in between the upper and lower lobe from where the middle lobe was previously resected. The anterior hilum is shown to be severely fibrosed and calcified.

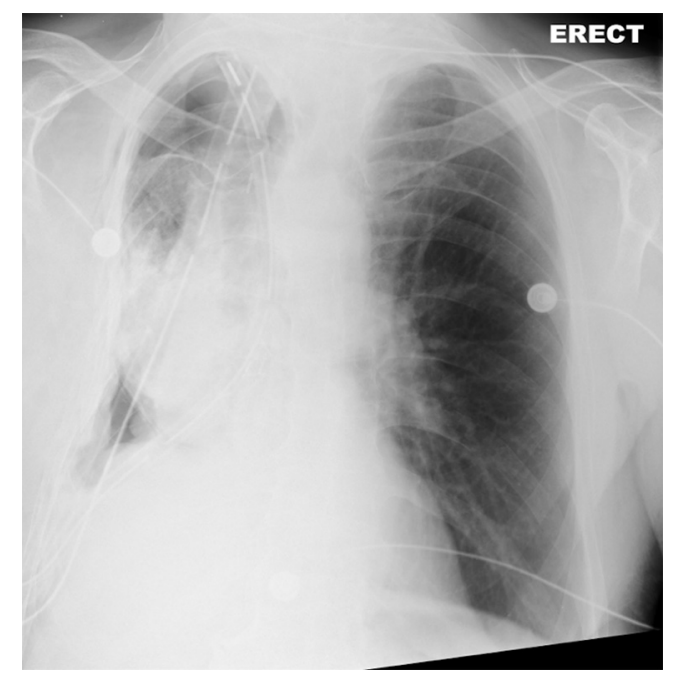

Figure 2 Chest X-ray obtained immediately postoperatively. The remaining right upper lobe is expanded but severely lacerated at its inferior aspect. There is also substantial pleural space.

endobronchial suctioning in a patient who after a complicated redo thoracotomy for lung cancer developed refractory collapse of the remaining upper lobe. This technique may be proven invaluable in the treatment of such demanding cases wherever the usual conservative measures fail. This article is presented in accordance with the CARE reporting checklist (available at http://dx.doi. org/10.21037/atm-20-3839).
Kouritas et al. Refractory post lung resection atelectasis

\section{Case presentation}

\section{Patient information}

A 70-year-old male was referred electively to our department for curative treatment of lung cancer with enlarging right lower lobe lesions.

Nine months before this referral the patient had undergone a right middle lobectomy for a similar cancer. This was performed via a double thoracotomy (entrance in chest via 2 thoracotomies but with one skin incision) which left a fluid-filled space between the upper and lower lobe (Figure 1).

The lesions were positron emission tomography (PET) avid (i.e., positive) and were proven on computed tomography (CT) guided biopsy to represent lung cancer (Figure 1).

His past medical history was significant mainly for ankylosing spondylitis with "bamboo spine" for which he was on methotrexate and steroids (20 mg/day). $\mathrm{He}$ also reported hypertension, history of bronchiectasis and previous pulmonary embolism (PE) for which he was on long-term anticoagulation. He was an ex-smoker with 40 pack years of smoking history.

His performance status was 0 but his lung function tests were borderline: Forced expiratory capacity in $1 \mathrm{sec}\left(\mathrm{FEV}_{1}\right)$ $=54 \%$ (1.2 liters) and Diffusional Capacity for Carbon Monoxide $(\mathrm{DLCO})=65 \%$. His cardiopulmonary exercise test showed an $\mathrm{MVO}_{2}=20.1(70.6 \%)$ and $\mathrm{VE} / \mathrm{VCO}_{2}=29$.

Apart from the lung multidisciplinary team (MDT) meeting, his case was discussed at the department's highrisk MDT and the decision was to proceed to a resection.

After a copious mediastinoscopy which was negative, he went on to have a redo right thoracotomy. Entrance in the chest was copious and demanding as the space was totally obliterated. Similarly, the hilum was fibrosed and access to the major vessels was eventually achieved intrapericardially. Based on the patient's co-morbidities, lung function and his long treatment with steroids, a pneumonectomy was decided to be avoided and therefore an intrapericardial lower lobectomy was performed. The remaining upper lobe re-expanded but its inferior surface was importantly lacerated (Figure 2).

\section{Clinical findings}

On postoperative day (POD) 1 the patient was well and 
Table 1 Timeline of events after redo-surgery (time point 0 )

\begin{tabular}{|c|c|c|c|c|c|c|c|c|c|c|}
\hline $\begin{array}{l}9 \text { months } \\
\text { before current } \\
\text { admission }\end{array}$ & $\begin{array}{l}\text { Time point } \\
\text { zero }(0)\end{array}$ & POD1 & POD2 & POD3 & POD4-5 & POD6 & POD7-8 & POD13 & POD40 & 4 months \\
\hline
\end{tabular}

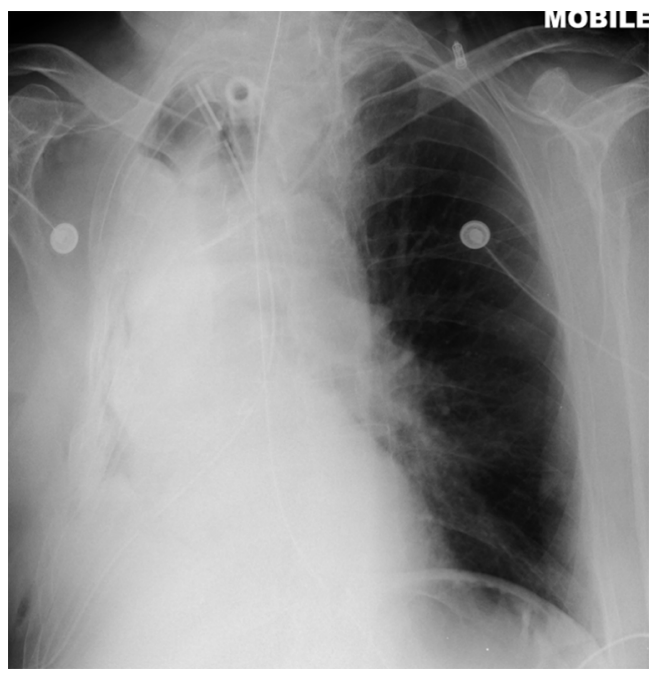

Figure 3 Chest X-ray obtained on postoperative day 1. A total atelectasis i.e., "white out" of the lung is noted.

received vigorous physiotherapy with mobilization and treatment with nebulizers (salbutamol $2.5 \mathrm{mgs}$ four times/ day, normal saline $0.9 \% 5 \mathrm{mLs}$ four times/day).

On POD 2 unfortunately he developed type 1 respiratory failure with increased oxygen requirements (from nasal cannula 3 litres to face mask $40 \%$ ). On examination, respiratory rate was elevated (30 breaths/min) and accessory breathing muscles were used. There were no breathing sounds heard on the operated side.

\section{Timeline}

The postoperative events are outlined in Table 1 .

\section{Diagnostic assessment}

On POD2 a totally occluded RUL i.e., a "white out"

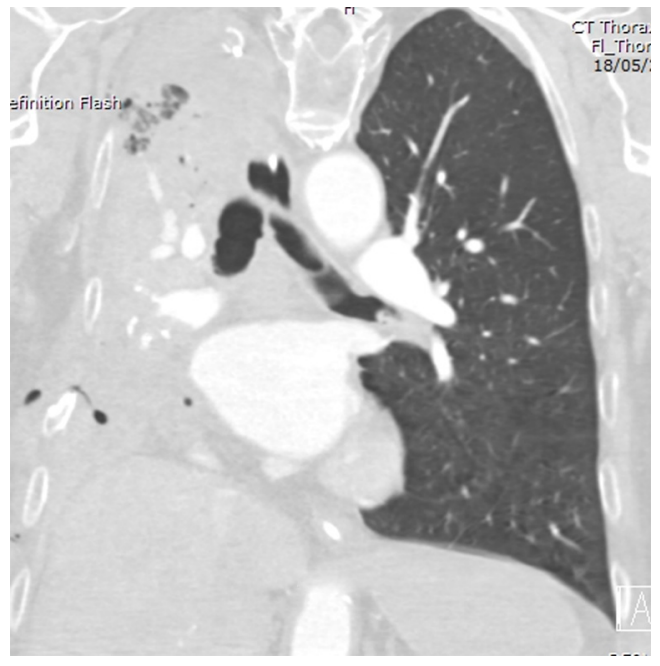

Figure $4 \mathrm{~A}$ CT chest of the patient was obtained on postoperative day 3 as the "white out" was refractory to any treatment up to that day. The scan showed totally atelectatic lung with some areas of aeration and patent main vessels.

(Figure 3) was found on his chest X-ray. Under the fear of lung torsion/infarction or a new massive PE, an urgent CT Chest was conducted on the same day, which showed patent vessels to the lobe and a totally atelectatic lung with mucous plugging of the main airways (Figure 4). His inflammatory markers got worse on that day: white blood cell count $(\mathrm{WBC})=23,100 \times 10^{9} / \mathrm{L}$ and $\mathrm{C}$-reactive protein $(\mathrm{CRP})=138 \mathrm{mg} / \mathrm{L}$.

The working diagnosis at this point therefore was respiratory failure/respiratory infection because of total atelectasis/pneumonia of the remaining RUL.

Failure to treat this sequeala would necessitate return to theatre for a high-risk pneumonectomy in terms of morbidity/mortality (i.e., bronchopleural fistula, empyema, urgent pneumonectomy in an infected space, longstanding 


\section{Page 4 of 6}

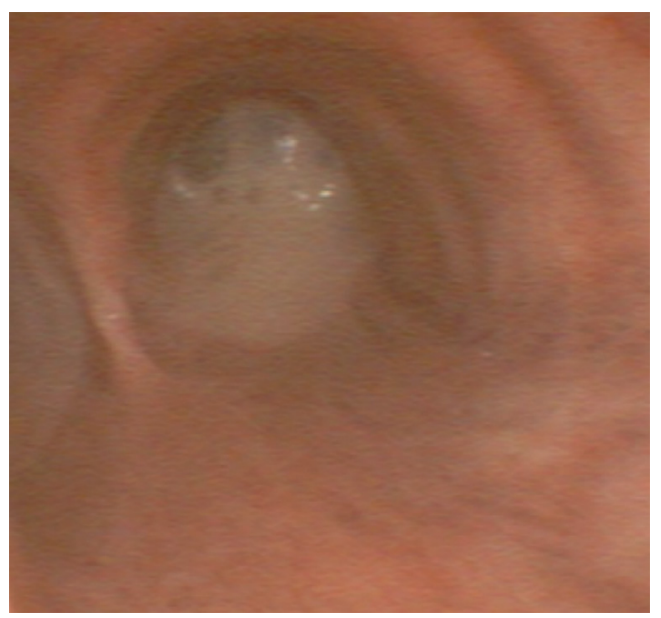

Figure 5 Repeated bronchoscopies showed thick and creamy secretions originating from the right main bronchus.

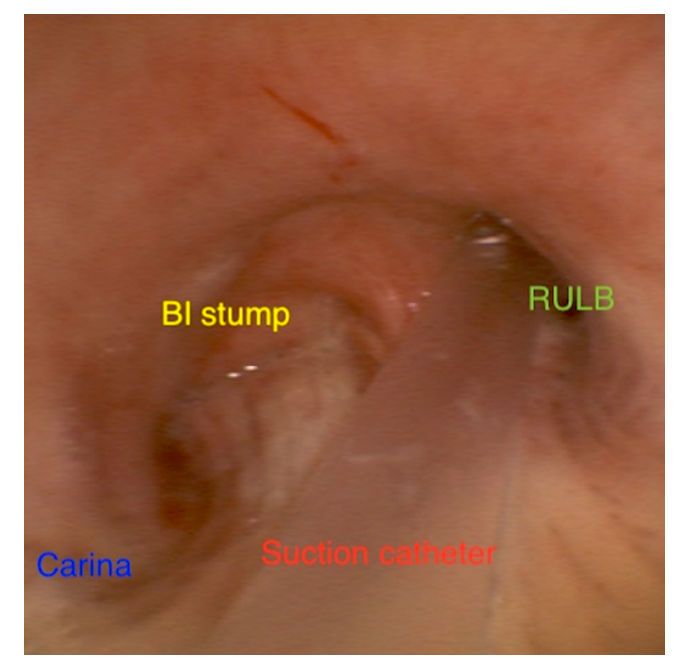

Figure 6 A suction catheter was left inside the right upper lobe bronchus orifice under bronchoscopic guidance. On this image, the bronchial intermedius stump and the carina are noted for reference.

lung infection/collapse, respiratory failure, intensive care unit stay).

\section{Therapeutic intervention}

Upon diagnosis, the patient was started on antibiotics (Piperacillin/Tazobactam 4.5 grs every 8 hours) and mucolytics (Carbocisteine $325 \mathrm{mg}$ every 8 hours) on top of his nebulizers.

\section{Kouritas et al. Refractory post lung resection atelectasis}

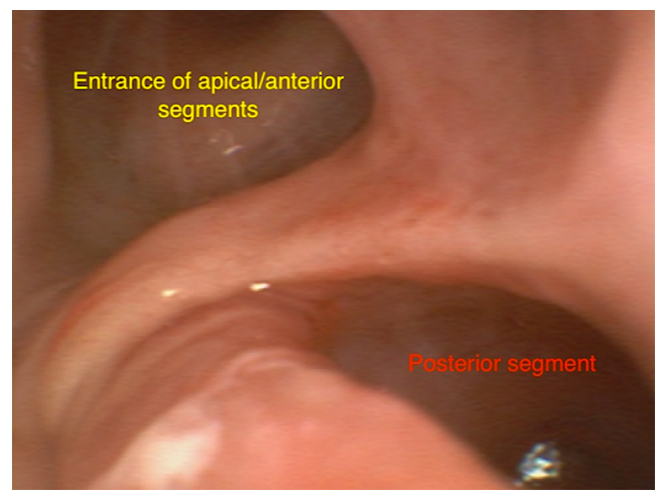

Figure 7 Clear orifice of the right upper lobe bronchus after removal of the catheter.

On POD 3, the patient returned to theatre for bronchoscopy. A tracheostomy (Size Fr 8.0) was additionally inserted.

Despite regular suctioning and vigorous physiotherapy for 2 days (POD 4 and 5) his "white out" did not improve. Additionally, the patient needed sedation for every bronchoscopy (as he could not tolerate it), his airways filled up with pus and creamy secretions almost immediately after every bronchoscopy (Figure 5) and blind suctioning through the tracheostomy was risky because of the bilobectomy bronchial stump.

On POD 6 and after all treatment measures have failed, returning to theatre for a high-risk pneumonectomy was the only choice left at this point.

As a last resort to avoid this high-risk reoperation/ completion pneumonectomy, a decision was made to sedate the patient and leave a suction catheter through his tracheostomy inside the RUL bronchus.

A suction catheter (16 Fr) was placed inside the RUL bronchus under bronchoscopic guidance (Figure 6) applying continuous endobronchial suction.

The rationale was to continuously provide suction to the infected lung, keeping airways clear in order to allow time for his lung to heal with antibiotics and steroids.

\section{Follow-up and outcomes}

The bronchoscopic picture (Figure 7), the "white out" on chest X-ray (Figure 8) and his inflammatory markers improved within the next 2 days (from POD 7) after which his sedation was discontinued.

He was discharged from ICU on POD 13 but he needed a long recovery (patient followed physiotherapy sessions), 


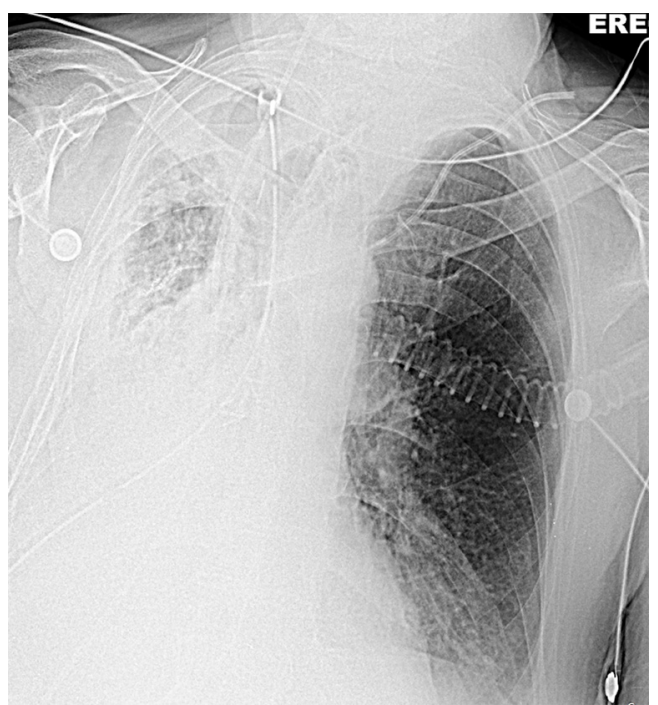

Figure 8 Chest X-ray obtained on the day the catheter was removed which shows aerated lung without a noticeable pleural space.

insertion of a long term drain for infected pleural effusion and was eventually discharged home a month later in good condition appreciating the outcome.

The patient unfortunately passed away a couple of months later because of type 2 respiratory failure.

\section{Helsinki declaration}

All procedures performed in studies involving human participants were in accordance with the ethical standards of the institutional and/or national research committee(s) and with the Helsinki Declaration (as revised in 2013). Informed consent was obtained from the patient.

\section{Discussion}

We present the technique of continuous endobronchial suctioning with a suction catheter left directly inside the RUL bronchus in a patient who developed a refractory atelectasis after complicated redo lung resection. This is the first time, to our knowledge, that such a technique is reported, and it may help physicians treating similar complicated situations in the future.

Lobar atelectasis postoperatively is a usual complication which can vary in severity (3). It could represent a simple atelectasis seen on a chest $\mathrm{X}$-ray requiring simple conservative treatment measures and physiotherapy or a severe pneumonia requiring, apart from antibiotic therapy, admission to a high dependency/intensive care unit. Bronchoscopy, preferably with a rigid bronchoscope +/tracheostomy are frequently required especially in cases with relapse $(6,7)$. In our case, the patient developed a total "white out" postoperatively not been able to be treated with usual conservative measures. Moreover, it was a complicated case as even back to back bronchoscopies were unsuccessful leading to quick re-accumulation of creamy secretions originating from the periphery of the remaining lung.

In this "uncharted" postoperative recovery situation we improvised by leaving the catheter inside the source of secretions i.e., the RUL bronchus (the remaining after the lower bilobectomy) leaving time for the infection to subside, keeping the airways and the bilobectomy bronchial stump clear and at the same time avoiding trauma to it from blind suctioning through the tracheostomy. Other authors have published such endeavors for example using DNase instillation in the airways or using double lumen endotracheal intubation for left lung atelectasis $(4,5)$. To our knowledge this practice of leaving a catheter inside the airways for continuous suctioning is reported for the first time.

Unfortunately, the patient passed away 4 months later from a non-cancer-related issue following the projection of lung resections in which when cardiopulmonary complications develop, patients accrue higher mortality (8).

Similarly, bilobectomies, against common belief, develop more cardiopulmonary complications rather than pleural space issues and this was verified with our case (9). Kinking of the airway of the remaining lobe because of his important collapse from his atelectasis/pneumonia could have been one of the factors that led to this ongoing lobe infection/ collapse. Continuous suctioning of the lobe possibly led to subsidence of the lobe inflammation and better expansion which consequently led to less airway kinking and better secretion clearance.

Ankylosis spondylitis is a disease which can surcharge negatively to the perioperative and postoperative period after surgery (10). In our case, the patient underwent an initial double thoracotomy (no reason recorded) and lymphadenectomy which, with this underlying disease, possibly induced a profound healing process ending up in extensive fibrosis inside the chest making the redoprocedure extremely copious.

One of the limitations of this technique was the inability to manage ventilation and achieve the desired airway 
pressures because of leakage around the catheter inside the tracheostomy, which necessitated extra maneuvers to accomplish a tight seal. Additionally, the patient had to be sedated instead of being awake and receiving physiotherapy, which however in our case, did not yield the desired results.

In summary, in cases with important refractory atelectasis with quick re-accumulation of secretions, continuous suctioning of the airways with a suction catheter inside the airway of issue, could potentially comprise another treatment modality for physicians who face this challenging complication/pathology.

\section{Acknowledgments}

Funding: None.

\section{Footnote}

Reporting Checklist: The authors have completed the CARE reporting checklist. Available at http://dx.doi.org/10.21037/ atm-20-3839

Conflicts of Interest: All authors have completed the ICMJE uniform disclosure form (available at http://dx.doi. org/10.21037/atm-20-3839). The authors have no conflicts of interest to declare.

Ethical Statement: The authors are accountable for all aspects of the work in ensuring that questions related to the accuracy or integrity of any part of the work are appropriately investigated and resolved. All procedures performed in studies involving human participants were in accordance with the ethical standards of the institutional and/or national research committee(s) and with the Helsinki Declaration (as revised in 2013). Informed consent was obtained from the patient.

Open Access Statement: This is an Open Access article distributed in accordance with the Creative Commons Attribution-NonCommercial-NoDerivs 4.0 International
License (CC BY-NC-ND 4.0), which permits the noncommercial replication and distribution of the article with the strict proviso that no changes or edits are made and the original work is properly cited (including links to both the formal publication through the relevant DOI and the license). See: https://creativecommons.org/licenses/by-nc-nd/4.0/.

\section{References}

1. Villeneuve PJ. Interventions to avoid pulmonary complications after lung resection. J Thorac Dis 2018;10:S3781-8.

2. Lugg ST, Agostini PJ, Tikka T, et al. Long-term impact of developing a postoperative pulmonary complication after lung surgery. Thorax 2016;71:171-6.

3. Marini JJ. Acute lobar atelectasis. Chest 2019;155:1049-58.

4. Assallum H, Song TY, DeLorenzo L, et al. Bronchoscopic instillation of DNase to manage refractory lobar atelectasis in a lung cancer patient. Ann Transl Med 2019;7:363.

5. Ozturk Z, Bayrakci B. A novel technique with double catheter suctioning for clearing away the left lung atelectases: Selective left bronchial aspiration (SeLBA). Clin Respir J 2019;13:728-32.

6. Massard G, Wihlm JM. Postoperative atelectasis. Chest Surg Clin N Am 1998;8:503-28.

7. $\mathrm{Wu} \mathrm{KH}$, Lin CF, Huang CJ, et al. Rigid ventilation bronchoscopy under general anesthesia for treatment of pediatric pulmonary atelectasis caused by pneumonia: A review of 33 cases. Int Surg 2006;91:291-4.

8. Lugg ST, Agostini PJ, Tikka T, et al. Long-term impact of developing a postoperative pulmonary complication after lung surgery. Thorax 2016;71:171-6.

9. Gómez MT, Jiménez MF, Aranda JL, et al. The risk of bilobectomy compared with lobectomy: a retrospective analysis of a series of matched cases and controls. Eur J Cardiothorac Surg 2014;46:72-5.

10. Blizzard DJ, Penrose CT, Sheets CZ, et al. Ankylosing Spondylitis Increases Perioperative and Postoperative Complications After Total Hip Arthroplasty. J Arthroplasty 2017;32:2474-9.
Cite this article as: Kouritas V, Ross N, Bilyy A, Hogan J, Parvez M, Kadlec J, Bartosik W, Van Tornout F. Continuous endobronchial suctioning for refractory post lobectomy lung atelectasis: a case report. Ann Transl Med 2021;9(9):815. doi: 10.21037/atm-20-3839 